\title{
Using the Resistance Depending on the Magnetic and Electric Susceptibility to Derive the Equation of the Critical Temperature
}

\author{
H. G. I. Hamza', M. H. M. Hilo², R. Abd Elgani'2, R. Abd Elhai'2, Mubarak Dirar² \\ ${ }^{1}$ Department of Physics, Gazera University, Hasahisa, Sudan \\ ${ }^{2}$ Department of Physics, Sudan University of Science and Technology, Khartoum, Sudan \\ Email: hasabo25@hotmail.com, mhhlo@qu.edu.sa, mahmoud1972@sustech.edu, rawia@sustech.edu, \\ rashaabdelhaye@sustech.edu
}

Received 23 September 2014; revised 20 October 2014; accepted 15 November 2014

Copyright (C) 2014 by authors and Scientific Research Publishing Inc.

This work is licensed under the Creative Commons Attribution International License (CC BY).

http://creativecommons.org/licenses/by/4.0/

(c) (i) Open Access

\begin{abstract}
In this study the electromagnetic theory and quantum mechanics are utilized to find the resistivity in terms of electric and magnetic susceptibility in which the electron is considered as a wave. Critical temperature of the wire at which the resistance vanishes is found. In this case the resistance being imaginary which leads the real part of the resistance to real zero at critical temperature and the material becomes super conductor in this case. If one considers the motion of electron in the presence of inner magnetic field and resistance force, a new formula for the conductivity is to be found; this formula states that the material under investigation becomes a superconductor at critical temperature and depends on the strength of the magnetic field and friction resistance, and the substance conductivity is found to be super at all temperatures beyond the critical temperature.
\end{abstract}

\section{Keywords}

Susceptibility, Superconductivity, Critical Temperature, Permeability, Permittivity

\section{Introduction}

In superconductors, the resistance is zero at temperatures less than the critical temperature [1]; in this work, the above concept is proved by taking the conductivity as a function of the permittivity and permeability, and within the existence of the conditions that make the resistance be equal to zero or approach to infinity. 


\section{Resistivity Formula Due to the Permeability and Permittivity}

When the temperature of a conductor approach to the absolute zero, the friction resistance can be ignored [2], if an electron e is induced by an electric field $E$, then the force on it is given by

$$
m \frac{\mathrm{d} v}{\mathrm{~d} t}=\mathrm{e} E
$$

Including the position variable $x$ in Equation (1) it can be written as

$$
m \frac{\mathrm{d} v}{\mathrm{~d} x} \frac{\mathrm{d} x}{\mathrm{~d} t}=\mathrm{e} E
$$

Then

$$
\int m v \mathrm{~d} v=\int \mathrm{e} E \mathrm{~d} x
$$

According to the definition of the potential $V$, we get

$$
E=-\frac{\mathrm{d} V}{\mathrm{~d} x}
$$

From Equation (3)

$$
\frac{m v^{2}}{2}=\mathrm{e} \int \frac{\mathrm{d} V}{\mathrm{~d} x} \mathrm{~d} x=\mathrm{e} V
$$

Then

$$
v=\frac{2 \mathrm{e} V}{m v}
$$

While $m$ is constant, and when the potential difference is constant, then the velocity $v$ is being also constant.

Using Equation (6) and substituting the value of $v$ in the equation of current, that given due to the electron velocity $v$, charges density $n$, and the area $A, I=n e v A$, then the current $I$ is found to be

$$
I=\frac{2 A n \mathrm{e}^{2} V}{m v}
$$

Then the resistance $R$ is given

$$
R=\frac{V}{I}=\frac{V}{n \mathrm{e} v A}=\frac{V m v}{A n \mathrm{e}[2 \mathrm{Ve}]}=\frac{m v}{2 A n \mathrm{e}^{2}}
$$

On other hand $R$ can be written due to the resistivity $\rho$, the length $l$, and the crossection area as

$$
R=\frac{\rho L}{A}
$$

Considering the electron as a wave, its velocity becomes [3]

$$
v=\frac{1}{\sqrt{\mu \varepsilon}}
$$

Accordingly the resistivity is given by

$$
\rho=\frac{m}{2 n \mathrm{e}^{2} L \sqrt{\mu \varepsilon}}
$$

\section{Critical Temperature at a Changing Permeability $\mu$}

If a magnetic field with a flux density $B$, an electric force $F_{e}$, besides a friction resistance $\gamma v$, and a pressure 
force $\nabla P=\nabla\left(\frac{1}{3} m n v^{2}\right)$ act together, then the centripetal forces which balance this force is given by [4].

$$
\frac{m v_{0}^{2}}{r}=B \mathrm{e} v_{0}+F_{e}-\gamma v_{0}-\frac{m}{3} v_{0}^{2} \nabla n
$$

where $v_{0}$ is the radial velocity, while the friction force and the pressure are given by

$$
F_{r}=\gamma v_{0}, \quad \nabla P=\frac{1}{3} m v^{2} \nabla n
$$

where $\gamma$ is the friction coefficient.

$$
m \omega_{0}^{2} r=B_{0} \mathrm{e} \omega_{0} r+F_{e}-\gamma \omega_{0} r-\frac{m}{3} \omega_{0}^{2} r^{2} \nabla n
$$

when the outer magnetic field vanishes, then the radial velocity becomes

$$
v_{0}=\omega_{0} r
$$

And

$$
F_{e}=m \omega_{0}^{2} r+B_{0} \mathrm{e} \omega_{0} r+\frac{m}{3} \omega_{0}^{2} r^{2} \nabla n
$$

where $B_{0}$ denotes the inner magnetic field.

And when an outer magnetic field $B$ is applied, then

$$
m \frac{\mathrm{d} v}{\mathrm{~d} t}=-\nabla P+F_{e}+F_{r}+\mathrm{e} B_{0} v+B \mathrm{e} v
$$

where $F_{r}$ is the radial force, and $F_{m}, F_{e}$ are the magnetic and the electric forces respectively, which are given by

$$
F_{B}=B e v \quad \text { and } \quad F_{e}=\mathrm{e} E
$$

The equation of motion in the presence of the outer magnetic field is given in the form [5].

$$
\frac{m v^{2}}{r}=-\frac{1}{3} m v^{2} \nabla n+F_{e}-\gamma v+B_{0} \mathrm{e} v+B \mathrm{e} v
$$

where $v$ is the radial velocity, and while $v=\omega r$ then

$$
\begin{aligned}
m \omega^{2} r & =-\frac{1}{3} m \omega^{2} r^{2} \nabla n+F_{e}-\gamma \omega r+B_{0} \mathrm{e} \omega r+B \mathrm{e} \omega r \\
& =-\frac{1}{3} m \omega^{2} r^{2} \nabla n+F_{e}-\gamma \omega r+B_{0} \mathrm{e} \omega r+B \mathrm{e} \omega r+m \omega_{0}^{2} r-B_{0} \mathrm{e} \omega r+\gamma \omega_{0} r+\frac{1}{3} \omega_{0}^{2} r^{2} \nabla n
\end{aligned}
$$

when $\omega$ is so closed to $\omega_{0}$ then

$$
\begin{gathered}
\omega \rightarrow \omega_{0} \text { and } \omega+\omega_{0}=2 \omega_{0} \\
\omega-\omega_{0}=\Delta \omega=\omega_{L}
\end{gathered}
$$

where $\omega_{L}$ is Larmar frequency, substitute Equation (15) and Equation (19) one gets?

$$
\begin{gathered}
m\left(\omega^{2}-\omega_{0}^{2}\right) r=-\frac{1}{3} m\left(\omega^{2}-\omega_{0}^{2}\right) r \nabla n-\gamma\left(\omega-\omega_{0}\right) r+B_{0} \mathrm{e}\left(\omega-\omega_{0}\right) r+B \mathrm{e} \omega r \\
m\left[1+\frac{r}{3} \nabla n\right] r\left(\omega-\omega_{0}\right)\left(\omega+\omega_{0}\right)=-r \gamma \omega_{L}+B_{0} \mathrm{e} \omega_{L} r+B \mathrm{e} \omega_{0} r \\
m\left[1+\frac{r}{3} \nabla n\right] r\left(2 \omega_{0}\right) \omega_{L}=-r \gamma \omega_{L}+B_{0} \mathrm{e} \omega_{L} r+B \mathrm{e} \omega_{0} r
\end{gathered}
$$

Dividing both sides by $\omega_{0} r$ we get 


$$
\begin{aligned}
& {\left[2 m\left[1+\frac{r}{3} \nabla n\right]+\frac{\gamma}{\omega_{0}}-\frac{B_{0} \mathrm{e}}{\omega_{0}}\right] \omega_{L}=B \mathrm{e}} \\
& \omega_{L}=\frac{\mathrm{e}}{\left[2 m\left[1+\frac{r}{3} \nabla n\right]+\frac{\gamma}{\omega_{0}}-\frac{B_{0} \mathrm{e}}{\omega_{0}}\right]} B
\end{aligned}
$$

The current for one atom with $Z$ electrons, moving around its nucleus with a frequency $f$ is

$$
i=+Z \mathrm{e} f=+\frac{Z \mathrm{e}}{2 \pi} \omega_{L}
$$

where $Z$ is the atomic number, e is the electron charge, and $\omega_{L}$ is Larmar frequency.

The magnetic torque for one atom is given by

$$
M_{a}=i A
$$

where $A$ is the area surrounded by the current which is equal

$$
A=\pi r_{e}^{2}
$$

And from Figure 1, one get:

$$
\begin{aligned}
& x=y=z \\
& r^{2}=x^{2}+y^{2}+z^{2} \\
& r^{2}=3 z^{2} \\
& \therefore z^{2}=\frac{1}{3} r^{2}
\end{aligned}
$$

But

$$
\begin{aligned}
r^{2} & =z^{2}+r_{e}^{2} \\
\therefore r^{2} & =\frac{1}{3} r^{2}+r_{e}^{2} \\
r_{e}^{2} & =\frac{2}{3} r^{2}
\end{aligned}
$$

So the magnetic torque for one atom $M_{a}$ becomes

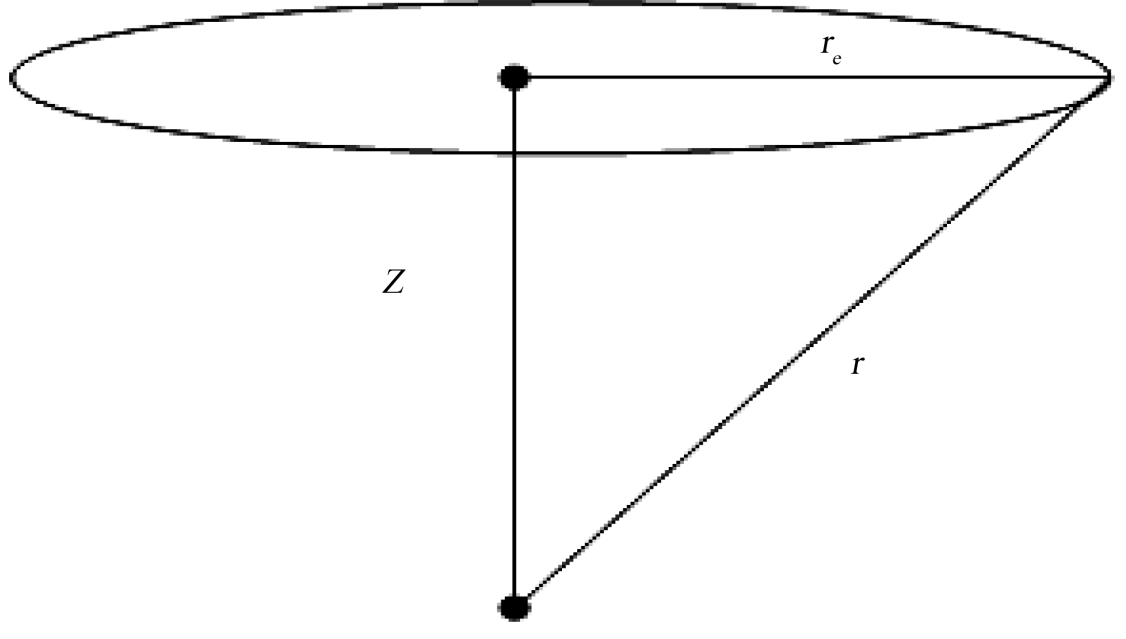

Figure 1. Magnetic torque in $Z$ direction. 


$$
M_{a}=\frac{2 \pi i r^{2}}{3}
$$

If the number of atoms per unit volume is assumed to be $N$ then, the magnetic torque for the matter is

$$
\begin{gathered}
M=N M_{a}=\frac{2 \pi}{3} N r^{2} \frac{z \mathrm{e} \omega_{L}}{2 \pi}=\frac{N z e r^{2}}{3} \omega_{L} \\
M=+\frac{N z r^{2} \mu_{0}}{3\left[2 m\left[1+\frac{r}{3} \nabla n\right]+\frac{\gamma}{\omega_{0}}-\frac{B_{0} \mathrm{e}}{\omega_{0}}\right]} H
\end{gathered}
$$

According to the definition of susceptibility $\chi_{m}$ then [6].

$$
M=\chi_{m} H
$$

Comparing Equations (26) and (27) the susceptibility being

$$
\chi_{m}=+\frac{N z r^{2} \mu_{0} \omega_{0}}{3\left[2 m\left[1+\frac{r}{3} \nabla n\right] \omega_{0}+\gamma-B_{0} \mathrm{e}\right]}
$$

Then the resistivity in Equation (11) becomes

$$
\rho=\frac{m}{2 n e L \sqrt{\varepsilon_{0} \mu}}=\frac{m}{2 n \mathrm{e} L \sqrt{\varepsilon_{0} \mu \frac{N z r^{2} \omega_{0}}{3\left[\frac{2 m}{\hbar}\left[1+\frac{r}{3} \nabla n\right] \frac{1}{2} k T+\gamma-B_{0} \mathrm{e}\right]}}}
$$

where $\hbar \omega_{0}=\frac{1}{2} k T$ denotes the photon energy.

The resistivity $\rho$ is imaginary, and the real resistivity vanishes when

$$
\begin{aligned}
& 3\left[\frac{2 m}{\hbar}\left[1+\frac{r}{3} \nabla n\right] \frac{1}{2} k T+\gamma-B_{0} \mathrm{e}\right] \leq 0 \\
& \text { or } 3\left[\frac{2 m}{\hbar}\left[1+\frac{r}{3} \nabla n\right] \frac{1}{2} k T\right] \leq B_{0} \mathrm{e}-\gamma
\end{aligned}
$$

Accordingly the critical temperature becomes

$$
T_{c}=\frac{2\left(B_{0} \mathrm{e}-\gamma\right)}{3\left[\frac{2 m}{\hbar}\left[1+\frac{r}{3} \nabla n\right]\right] k}
$$

\section{Calculating the Critical Temperature Due to the Conductivity}

Assuming that the charges in the conductor are acted by a resistance force $F_{r}$, and a magnetic force $F_{m}$, besides the electric force $F_{e}$, and then the equation of motion becomes [7].

$$
F=F_{r}+F_{m}+F_{e}
$$

The previous forces are given by the formulas

$$
\begin{array}{ll}
F_{0}=k_{0} x, & F_{r}=\frac{n m v}{\tau} \\
F_{m}=B \mathrm{e} v, & F_{e}=\mathrm{e} E
\end{array}
$$

where $n, k, x, m, v, \mathrm{e}, B, \tau$ and $E$ denotes the density, rigidity coefficient, displacement, mass, velocity, 
electron charge, magnetic flux density, resolving time, and the electric field respectively.

The equation of motion takes the formula

$$
m a=\frac{n m v}{\tau}+B \mathrm{e} v+\mathrm{e} E
$$

When the electron moves with a uniform constant velocity, the Equation (33) becomes

$$
\left(\frac{m n}{\tau}-B \mathrm{e}\right) v=\mathrm{e} E, \quad v=\frac{\mathrm{e}}{\left(\frac{m n}{\tau}-B \mathrm{e}\right)} E
$$

And the conductivity is given by

$$
J=n_{e} \mathrm{e} v=\frac{n_{e} \mathrm{e}^{2}}{\left(\frac{n m}{\tau}-B \mathrm{e}\right)} E
$$

where $n_{e}$ the electrons density, while $n$ denotes the density of the medium atoms, accordingly the conductivity being

$$
\sigma=\frac{n_{e} \mathrm{e}^{2}}{\left(\frac{n m}{\tau}-B \mathrm{e}\right)} E=\sigma E
$$

And the conductivity approaches to infinity when

$$
\frac{n m}{\tau}-B e=0
$$

According to the Maxwell-Boltzmann statistics the density of the atoms in the medium takes the formula [8].

$$
n=n_{e} \mathrm{e}^{-\frac{E}{k T}} \approx n_{0}\left(1-\frac{E}{k T}\right)
$$

Then

$$
\begin{gathered}
\frac{m n_{0}}{\tau}\left(1-\frac{E}{k T}\right)=B \mathrm{e} \\
\frac{E}{k T}=1-\frac{B \mathrm{e} \tau}{m n_{0}}=\frac{m n_{0}-B \mathrm{e} \tau}{m n_{0}} \\
T_{c}=\frac{m n_{0} E}{k\left(m n_{0} B \mathrm{e} \tau\right)}
\end{gathered}
$$

Equation (38) represents the critical temperature in which the conductivity becomes very huge, and when

$$
\frac{n m}{\tau}-B e \ll 1
$$

The conductivity also becomes very high, and then

$$
\begin{gathered}
\frac{n m}{\tau}\left[1-\frac{E}{k T}\right]-B \mathrm{e} \ll 1 \\
1-\frac{E}{k T} \ll \frac{B \mathrm{e} \tau}{m n_{0}} \\
-\frac{E}{k T} \ll \frac{B \mathrm{e} \tau}{m n_{0}}-1
\end{gathered}
$$




$$
\frac{E}{k\left(1-\frac{B \mathrm{e} \tau}{m n_{0}}\right)} \gg T
$$

And finally the critical temperature is found to be

$$
T_{c}=\frac{m n_{0} E}{k\left(m n_{0}-B e \tau\right)}
$$

\section{Discussion}

The classical rules of the electron motion in Equation (1) are used to find the classical formula of the resistivity given in Equation (11), and the electron is considered to be a wave according to the quantum principles and this clarified that the resistivity is a function of the electric and magnetic susceptibility.

The interpretation of Equation (28) - in which we derived the magnetic susceptibility from the electron equation of motion, that depend on the friction force within the friction coefficient $\gamma$, the inner magnetic field $B_{0}$, the grad of the electrons density $\nabla n$, and the atom radius $r$-is that when Equation (28) is substituted in Equation (11) the critical temperature at which the resistance vanishes, was found to be in the form that given by Equation (31), which was completely depends on the inner magnetic field $B_{0}$, and the friction, within the coefficient $\gamma$, the radius $r$, and the grad of the electron density $\nabla n$.

When we considered the electron motion due to the impact of an inner magnetic field, and a friction resistance, the conductivity was found to be as shown in Equation (36).

The mathematical analysis interprets that the conductivity becomes very high at temperatures less than the critical temperature, which depends on the friction resistance and the inner magnetic field as shown in Equations (41)-(42).

\section{Conclusion}

The model in which the resistance depends on the electric and magnetic susceptibility, clarifies that the resistance vanishes, and the metal becomes a superconductor at the critical temperature and the temperatures less than it; this relation is not clear in the famous models of the superconductivity.

\section{References}

[1] Sales, B.C., et al. (2012) Transport, Thermal, and Magnetic Properties of the Narrow Gap Semiconductor CrSb ${ }_{2}$. Physical Review B, 86, Article ID: 235136. http://dx.doi.org/10.1103/PhysRevB.86.235136

[2] Nguyen, D.N., et al. (2009) Temperature Dependence of Total AC Loss in High-Temperature Superconducting Tapes. IEEE Transactions on Applied Superconductivity, 19, 3637-3644.

[3] Slooten, E., et al. (2009) Enhancement of Superconductivity near the Ferromagnetic Quantum Critical Point in UCoGe. Physical Review Letters, 103, Article ID: 097003.

[4] Millican, J.N., Phelan, D., Thomas, E.L., Leao, J.B. and Carpenter, E. (2009) Solid State Communications, $149,707$.

[5] Kantorovich, L. (2004) Quantum Theory of the Solid State: An Introduction. Kluwer Academic Publishers, London. http://dx.doi.org/10.1007/978-1-4020-2154-1

[6] Weyeneth, S., Puzniak, R., Mosele, U., Zhigadlo, N.D., Katrych, S., Bukowski, Z., Karpinski, J., Kohout, S., Roos, J. and Keller, H. (2009) Anisotropy of Superconducting Single Crystal $\mathrm{SmFeAsO}_{0.8} \mathrm{~F}_{0.2}$ Studied by Torque Magnetometry. Journal of Superconductivity and Novel Magnetism, 22, 325-329. http://dx.doi.org/10.1007/s10948-008-0413-1

[7] Weyeneth, S., Puzniak, R., Zhigadlo, N.D., Katrych, S., Bukowski, Z., Karpinski, J. and Keller, H.J. (2009) Evidence for Two Distinct Anisotropies in the Oxypnictide Superconductors $\mathrm{SmFeAsO}_{0.8} \mathrm{~F}_{0.2}$ and $\mathrm{NdFeAsO}_{0.8} \mathrm{~F}_{0.2}$. Journal of Superconductivity and Novel Magnetism, 22, 347-351. http://dx.doi.org/10.1007/s10948-009-0445-1

[8] De Visser, A., et al. (2009) Muon Spin Rotation and Relaxation in the Superconducting Ferromagnet UCoGe. Physical Review Letters, 102, Article ID: 167003. http://dx.doi.org/10.1103/PhysRevLett.102.167003 
Scientific Research Publishing (SCIRP) is one of the largest Open Access journal publishers. It is currently publishing more than 200 open access, online, peer-reviewed journals covering a wide range of academic disciplines. SCIRP serves the worldwide academic communities and contributes to the progress and application of science with its publication.

Other selected journals from SCIRP are listed as below. Submit your manuscript to us via either submit@scirp.org or Online Submission Portal.
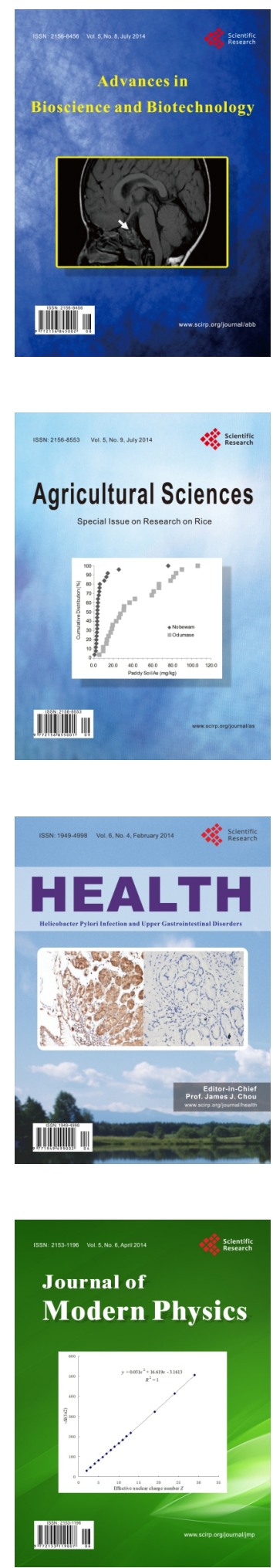
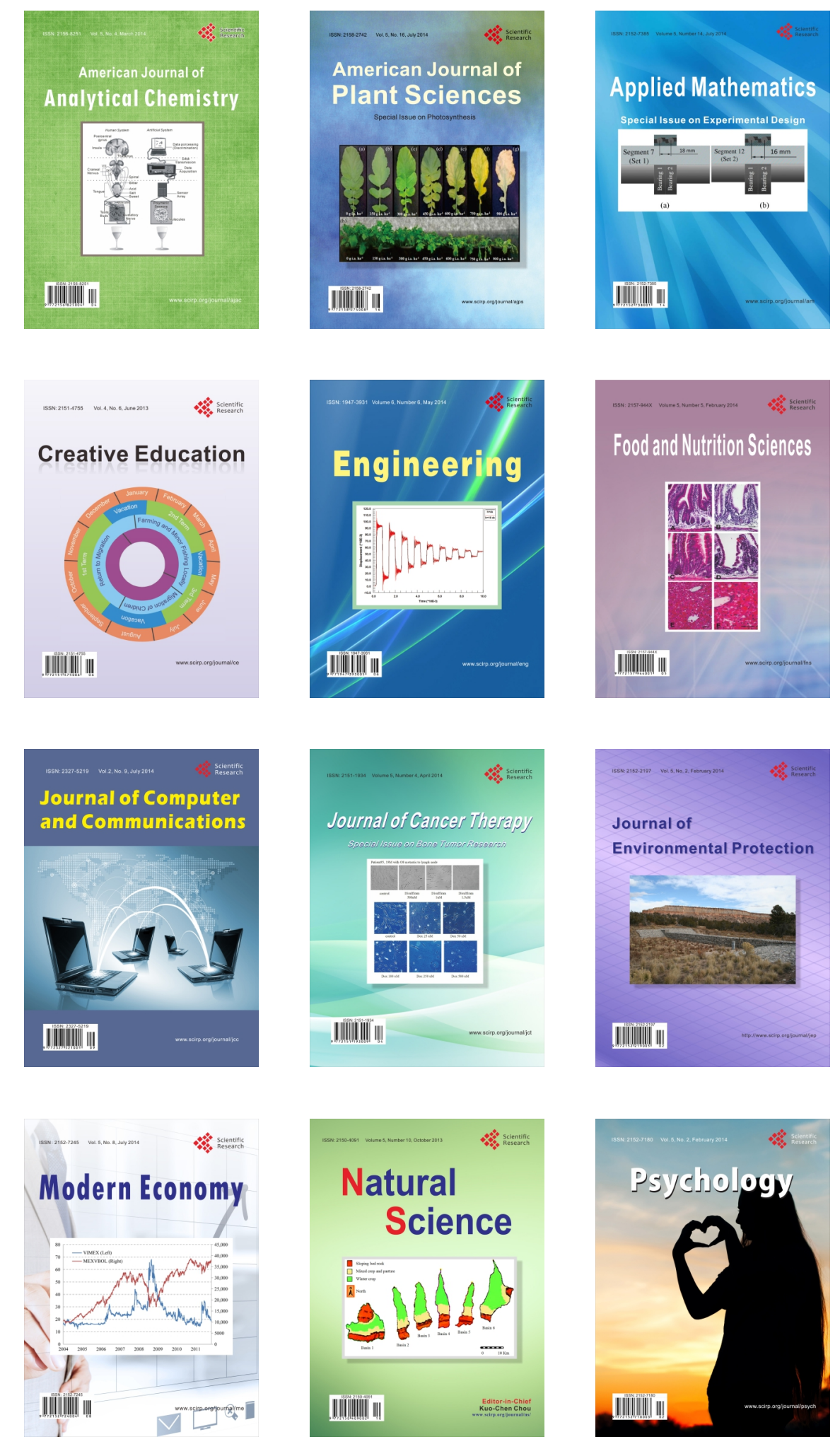